\title{
Metschnikowia bicuspidata and Enterococcus faecium co-infection in the giant freshwater prawn Macrobrachium rosenbergii
}

\author{
Shih-Chu Chen ${ }^{1, *}$, Tung-Hung Chen ${ }^{1}$, Pei-Chi Wang ${ }^{1}$, Yu-Chin Chen ${ }^{1}$, Ju-Ping Huang ${ }^{1}$, \\ Yu-De Lin ${ }^{1}$, Hso-Chi Chaung ${ }^{1}$, Li-Ling Liaw ${ }^{2}$
}

${ }^{1}$ Department of Veterinary Medicine, National Pingtung University of Science and Technology, Pingtung 912, Taiwan, ROC

${ }^{2}$ Bioresources Collection and Research Center, Food Industry Research and Development Institute, Hsinchu 300, Taiwan, ROC

\begin{abstract}
In May 2001, an epizootic yeast and bacterial co-infection in the giant freshwater prawn Macrobrachium rosenbergii occurred in Taiwan causing a cumulative mortality of $25 \%$. The diseased prawns had a yellowish-brown body color, milky hemolymph, opaque, whitish muscles, and were approximately 7 mo old with total lengths ranging from 8 to $10 \mathrm{~cm}$. Histopathological examination showed marked edema, yeast infiltration, and necrotic lesions with inflammation in the muscles, hepatopancreas and other internal organs. We isolated 2 pathogens from the diseased prawns, one was a yeast (AOD081MB) and the other a Gram-positive coccus (AOD081EF). The Gram-positive coccus was identified as Enterococcus faecium by the API 20 Strepsystem, conventional biochemical tests, and it had $99 \%$ 16S rDNA sequence identity (GenBank Accession Number AJ276355) to E. faecium (GenBank Accession Number AF529204). The sequence of a PCR product from the D1/D2 domain of 26S rDNA (GenBank Accession Number AF529297) from the yeast gave $99 \%$ sequence identity to Metschnikowia bicuspidata (GenBank Accession Number U44822). Experimental infections with these isolates produced gross signs and histopathological changes similar to those observed in the naturally infected prawns. The lethal doses $\left(\mathrm{LD}_{50}\right)$ for isolate E. faecium AOD081EF, $M$. bicuspidata AOD081MB and the co-infection were $4.7 \times 10^{4}, 2.6 \times 10^{2}$, and $2.4 \times 10^{2}$ colony-forming units prawn ${ }^{-1}$, respectively. This is the first report of a confirmed co-infection of $M$. bicuspidata and E. faecium in prawn aquaculture.
\end{abstract}

KEY WORDS: Metschnikowia bicuspidata - Enterococcus faecium · Giant freshwater prawn · Macrobrachium rosenbergii $\cdot$ PCR

\section{INTRODUCTION}

The giant freshwater prawn Macrobrachium rosenbergii is commercially cultured throughout the world, and intensively so in Taiwan, where production decreased by 47 to $52 \%$ from 1992 to 1995 (New 1995) due to disease problems. There are 2 diseases that have been linked to production decline. One is a yeast infection that occurs mostly in the cool season (October to March), with clinical signs including a yellow exoskeleton, a swollen hepatopancreas (HP), milky hemolymph and opaque, whitish muscles (Cheng \&
Chen 1998a, Lu et al. 1998). The other is an Enteroccoccus-like infection that occurs mostly in the hot season (June to September, especially during phytoplankton blooms) and causes muscle necrosis (Cheng \& Chen 1998a,b). Recent studies have indicated that mortality in $M$. rosenbergii caused by this bacterium is exacerbated by suboptimal environmental temperatures and $\mathrm{pH}$ (Cheng \& Chen 1998c). An additional further study identified the causative bacterium as Lactococcus garvieae by PCR assay and by $16 \mathrm{~S}$ rDNA sequencing (Chen et al. 2001). Disease outbreaks of yeast among freshwater prawns have continued in 
southern Taiwan since 1988 and from 1994; most infections (64\%: Lu et al. 1998) have occurred during the winter (December to February). Prevalence has been highest in adult prawns (73\%), followed by juveniles $(25 \%)$ and postlarvae (2\%). No yeast infections have been found in larvae. Yeasts that are pathogenic for $M$. rosenbergii include Candida sake (I and II), Pichia anomala, Endomyces fibuliger and C. famata (Lu et al. 1998). Yeasts are often identified based on cellular morphology and distinctive reactions in standardized fermentation and assimilation tests. However, genetic crosses and molecular comparisons have shown that many of these phenotypic characteristics are strainspecific and inadequate for recognition of either species or genera. The D1/D2 domain of the 26S rDNA gene is sufficiently variable to allow reliable separation of all known and clinically significant yeast species (Kurtzman \& Robnett 1997). In this study, 2 pathogens from co-infections were identified as Enterococcus faecium and Metschnikowia bicuspidata by fermentation tests and molecular methods.

\section{MATERIALS AND METHODS}

Prawn samples. Diseased prawn samples (body weight 5 to $8 \mathrm{~g}$, length 8 to $10 \mathrm{~cm}$ ) were collected from 4 prawn farms during disease outbreaks in May 2001 for histopathological and microbiological analyses; 5 prawns were sampled for this study from each farm. Prawn losses were as high as $48 \%$ in some ponds, and average losses were approximately $25 \%$. The prawns had been fed with commercial pellets (prawn diet, Gold Brand Feed).

Microbiology. The cuticle of each diseased prawn was surface-sterilized by swabbing with $70 \%$ (v/v) ethanol before cutting open to reveal underlying tissues. Muscle tissues and the hepatopancreas (HP) were swabbed and streaked onto tryptic soy agar (TSA; Difco, Becton Dickinson), blood agar $\left(\mathrm{BA}_{i}=\right.$ TSA $+5 \%$ goat blood), brain heart infusion agar (BHI, Difco), and Lowenstein-Jensen medium (LJM). Inoculated plates were incubated at $25^{\circ} \mathrm{C}$ for $7 \mathrm{~d}$ and single colonies of different size and color were re-streaked on the same media to obtain pure isolates. These isolates were identified using the API Strepsystem (bioMérieux $\mathrm{SA}$ ) and conventional bacteriological tests (Chen et al. 2001).

Pathology. Muscle tissue, hepatopancreas tissue and tissues of other internal organs with lesions were preserved in Davidson's fixative and processed for standard paraffin-sectioning. Sections were stained using haematoxylin and eosin (H\&E), Gram stain and periodic acid-Schiff (PAS) for viewing with a light microscope (Lu et al. 1998, Chen et al. 2001).
Bacterial strains. The following bacterial strains were used as controls for PCR assays: Lactococcus garvieae (American Type Culture Collection, ATCC 43921) and L. garvieae from rainbow trout (MZ 9101 [KG-] kindly provided by Dr. Terutoyo Yoshida, Miyazaki University, Japan). In addition, the following strains were obtained from the Culture Collection Research Center (CCRC), Taiwan: Enterococcus faecium CCRC 10067, E. faecalis CCRC 10066, E. durans CCRC 10790, E. avium CCRC 10801, L. lactis ssp. lactis CCRC 10791, L. lactis subspecies cremoris CCRC 11067, L. raffinolactis CCRC 14039, Metschnikowia bicuspidata CCRC 21466, and L. garvieae MR1 (the latter isolated from Macrobrachium rosenbergii; Chen et al. 2001). Two purified isolates derived from infected prawns in this study were labeled AOD081MB (yeast) and AOD081EF (cocci).

DNA isolation, PCR, and sequencing of $16 \mathrm{~S}$ rDNA for Coccus AOD081EF. PCR amplification of the 16S rDNA gene from Coccus AOD081EF was accomplished using purified DNA template with the Forward Primer 5F (5' TGG AGA GTT TCC TGG CTC AG 3') and the Reverse Primer 1540R (5' AAG GAG GTG ATC CAR CCG CA 3'). The PCR protocol was initial denaturation for $10 \mathrm{~min}$ at $95^{\circ} \mathrm{C}$ followed by 30 cycles of denaturation for $30 \mathrm{~s}$ at $95^{\circ} \mathrm{C}$, annealing for $30 \mathrm{~s}$ at $60^{\circ} \mathrm{C}$ and extension for $45 \mathrm{~s}$ at $72^{\circ} \mathrm{C}$. Final extension was at $72^{\circ} \mathrm{C}$ for $10 \mathrm{~min}$. The PCR product was purified with a QIAquick PCR purification kit (Qiagen). Sequencing of the 16S rDNA PCR fragment from Strain AOD081EF was carried out using a MicroSeq $^{\mathrm{TM}}$ 16S rRNA Gene Kit manufactured by Applied Biosystems and following the manufacturer's instructions. Sequences were determined using a Model 310 automatic DNA sequencer with Microse$\mathrm{q}^{\mathrm{TM}}$ software (Applied Biosystems). The resultant 16S rDNA sequence was manually aligned with sequences of representative strains retrieved from GenBank, DDBJ and EMBL databases (Chen et al. 2001).

DNA isolation, PCR, and sequencing of D1/D2 domain of Yeast AOD081MB 26S rDNA. The divergent domain at D1/D2 of the 26S rDNA gene from Yeast AOD081MB was symmetrically amplified with primers NL-1 (5' GCA TAT CAA TAA GCG GAG GAA AAG 3') and NL-4 (5' GGT CCG TGT TTC AAG ACG G 3') (Kurtzman \& Robnett 1997). The amplification protocol was 36 cycles with annealing at $52^{\circ} \mathrm{C}$ for $1 \mathrm{~min}$, extension at $72^{\circ} \mathrm{C}$ for $2 \mathrm{~min}$ and denaturation at $94^{\circ} \mathrm{C}$ for $1 \mathrm{~min}$. Both strands of the PCR amplicons were sequenced using an ABI Taq DyeDeoxy Terminator cycle sequencing kit (Applied Biosystems); 4 sequencing reactions were required for each DNA sample using the External Primers NL-1 and NL-4 and the Internal Primers NL-2A (5' CTT GTT CGC 
TAT CGG TCT C $3^{\prime}$ ) and NL-3A (5' GAG ACC GAT AGC GAA CAA G 3') (Kurtzman \& Robnett, 1997). PCR products $(5 \mu \mathrm{ll})$ underwent electrophoresis (35 min, $100 \mathrm{~V}$ ) in $2 \%$ agarose gel (SeaKem ${ }^{\circledR}$ LE agarose) with 1X TBE buffer (BDH Laboratory supplies) containing $90 \mathrm{mM}$ Tris, $90 \mathrm{mM}$ borate and $2 \mathrm{mM}$ EDTA (pH 8.3) and were visualized by ethidium bromide staining. The DNA molecular weight marker comprised a 100 bp ladder (100 bp ET marker, Chenig Hsin Tang Chemical).

Experimental infections. Healthy prawns (Macrobrachium rosenbergii of 5 to $7 \mathrm{~g}$ body weight) were obtained from a prawn farm in Pingtung, Taiwan, and were held at a density of 320 prawns in 3 continuously aerated $400 \mathrm{l}$ aquaria containing $300 \mathrm{l}$ of freshwater at approximately $27^{\circ} \mathrm{C}$ for $7 \mathrm{~d}$, until the prawns were acclimated to laboratory conditions. They were fed 2 times daily with a commercial prawn diet (Prawn diet, Gold Brand Feed). Waste from each tank was removed daily. Subsequently, 220 prawns of uniform size were subdivided into 22 groups of 10 prawns each. Serial 10 -fold dilutions from microbial turbidity $(\mathrm{OD}=$ 1 under $620 \mathrm{~nm}$ ) were prepared in sterile normal saline $(0.85 \% \mathrm{NaCl})$ from the purified pathogen isolates AOD081EF and AOD081MB. A dose of $0.1 \mathrm{ml}$ for each dilution was injected intramuscularly (i.m.) into 10 prawns of each treatment group (Groups 1 to 7 for Strain AOD081EF, 8 to 14 for AOD081MB and 15 to 21 for AOD081EF plus AOD081MB). Sterile normal saline $0.1 \mathrm{ml}$ was injected i.m. into 10 prawns of control Group 22. After injection, each group was maintained separately in an $80 \mathrm{l}$ aquarium under the same conditions as during the acclimation period. The prawns were continuously monitored for morbidity and mortality and sampled for histopathology and microbiology.

\section{RESULTS}

\section{Clinical signs and gross pathology of diseased prawns from farms}

Most moribund prawns had a history of poor growth, with clinical signs of anorexia, a yellowish-brown body color, swelling between the cephalothrax and abdomen (Fig. 1), a swollen HP, milky hemolymph and opaque, whitish muscle tissue. Yellowish or milky hemolymph was present when the cephalothorax was removed or when the muscle was cut during necropsy. Whitish-yellow spots of varying size $(0.5$ to $2 \mathrm{~cm})$ were also found on the surface and within muscle tissue. Smears from milky hemolymph, epidermis, muscle and HP showed a variety of yeast growth stages from 0.3 to $0.6 \mu \mathrm{m}$ in diameter (Fig. 2).

\section{Microbiology of diseased prawns from farms}

Bacterial colonies in samples from the muscle and HP appeared 1 to $2 \mathrm{~d}$ after culture on TSA, BA, and BHI. Microbial growth on agar plates with cultures from prawns with significant pathological lesions was higher than for cultures originating from prawns with slighter pathological lesions. This $10^{9}$ microbial cell growth in $\mathrm{ml}^{-1}$ hemolymph harvested from severely diseased prawn was counted by serial 10 -fold dilution. Two different-sized colonies were present after incubation on BHI agar: one type was initially small and transparent at $24 \mathrm{~h}$, but had grown to $0.1-0.3 \mathrm{~cm}$ in diameter and became opaque or milky-white after continued incubation to $48 \mathrm{~h}$; the other type began as a pinpoint-sized colony at $24 \mathrm{~h}$, and had grown to $0.05-0.1 \mathrm{~cm}$ in diameter and become semitransparent by $48 \mathrm{~h}$. Cells from both colony types were Gram-positive, but those from the large colonies had a typical yeast form $(0.3$ to $0.6 \mu \mathrm{m})$ while those from the small colonies were coccoid ( 0.1 to $0.3 \mu \mathrm{m})$ under the light microscope. Biochemical and physiological characteristics of representative isolates of the Gram-positive cocci (AOD081EF) are given in Table 1 and compared to reference isolates of Enterococcus spp. and Lactococcus spp.

\section{Histopathology of diseased prawns from farms}

In moribund prawns, edematous fluid accumulated between the cuticle and muscle and large numbers of yeast cells infiltrated the cuticle, epidermis and dermis. Marked fragmentation of cardiac fibers and edema were observed with hemocyte nodules in the heart (Fig. 3). In the HP, tubule epithelial cells were markedly vacuolated and the sinuses contained massive yeast clumps encapsulated by a thin membrane (Fig. 4). Large necrotic foci with yeast clumps and cellular debris were also observed. Desquamation of HP tubule epithelial cells producing mixed cellular debris and yeast cells was also seen in some prawns. Abdominal, pereiopod and pleopod muscles showed much edema and liquefactive necrosis, with large numbers of yeast cells (Figs. $5 \& 6$ ). The gills were also necrotic, with dilation and infarction of the capillaries and large numbers of yeast cells or yeast clumps.

\section{Sequence of 16S rRNA PCR amplicon of Coccus AOD081EF}

The 16S rDNA sequence of the coccus isolate AOD081EF is deposited at GenBank (accession number AF529204). It showed an identity of 99\% 

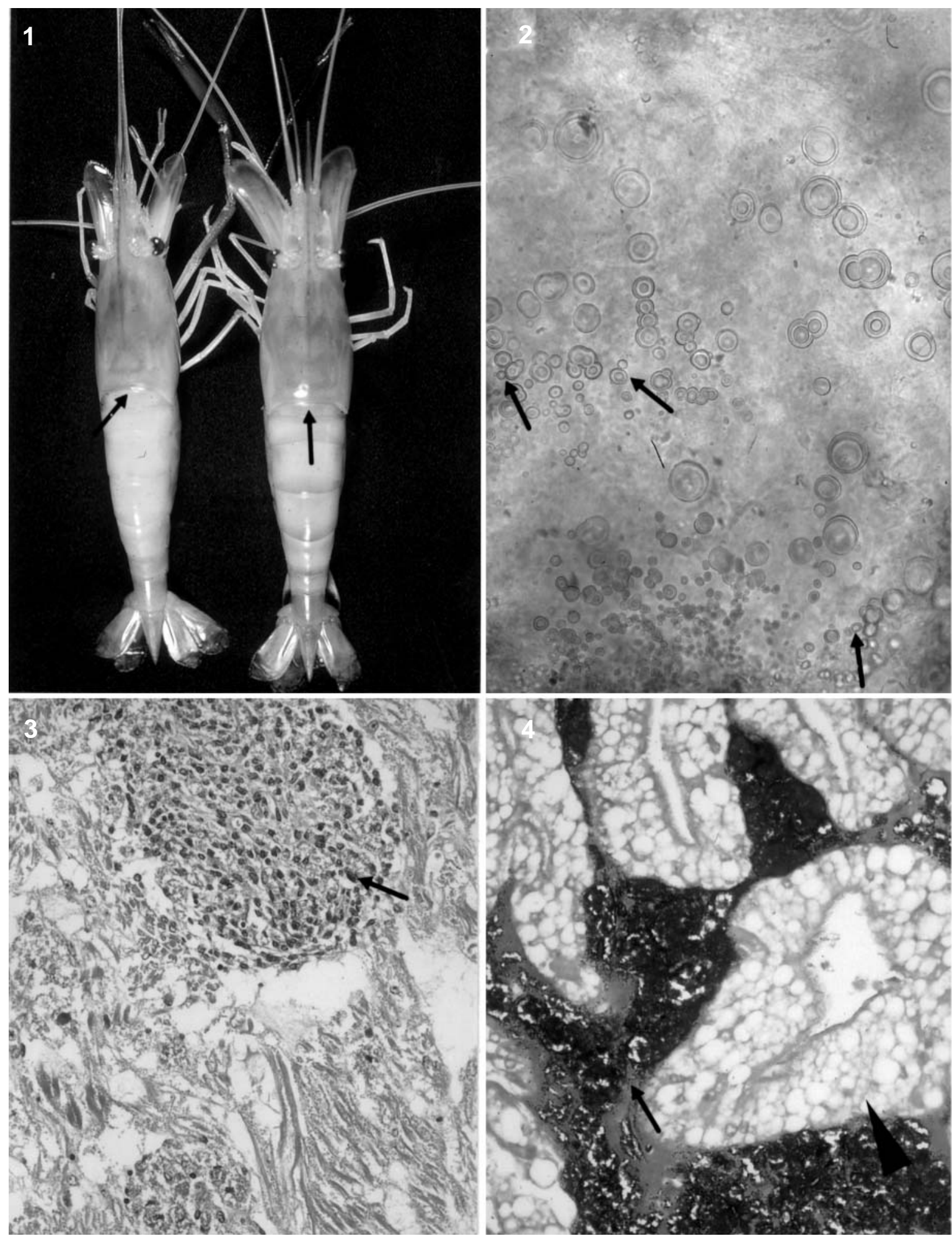

Figs. 1 to 4. Macrobranchium rosenbergii. Fig. 1. Giant freshwater prawns with swollen cephalothorax and expanded zone (arrows) in dorsal area between cephalothorax and first abdominal segment. Fig. 2. Wet mount from dermal tissue of a moribund prawn showing different growth stages of yeast (arrows) $(\times 200)$. Fig. 3. Tissue section of infected giant freshwater prawn with marked fragmentation of cardiac fibers and a haemocytic nodule in the heart (arrow) (H\&E, ×400). Fig. 4. Infected prawn hepatopancreas section showing vacuolation of hepatopancreas tubular epithelial cells (arrowhead) and yeast microcolonies (arrow) in the sinus (PAS, $\times 200)$ 
with Enterococcus faecium (EMBL AJ276355), 98\% with E. duran (EMBL AJ276354), 98\% with E. hirae (EMBL AJ276356), $97 \%$ with E. avium (GenBank, accession number AF061008) and $97 \%$ with E. raffinosus (EMBL Y18296).

\section{Sequence of 26S rDNA PCR amplicon of Yeast AOD081MB}

A representative agarose electrophoresis gel of PCR amplicons from the 26S rDNA gene of the yeast isolate AOD081MB is shown in Fig. 7. The pattern of 550 and $300 \mathrm{bp}$ amplicons for the first- and second-step PCR, respectively, was identical to that for Reference Strain Metschnikowia bicuspidata CCRC 21466 (data not shown). PCR results for bacteria including Entrococcus faecalis CCRC 10066, E. durans CCRC 10790, Lactococcus lactis ssp. lactis CCRC10791, L. lactis ssp. cremoris CCRC 11067, L. raffinolactis CCRC 14039, and E. avium CCRC 10801 were negative. The sequence of the D1/D2 domain of the 26S rDNA gene from our yeast isolate AOD081MB is deposited at GenBank (accession number AF529297). It shows an identity of $99 \%$ with $M$. bicuspidata (GenBank U44822), $94 \%$ with M. australis (GenBank U76526), 90\% with M. reukaufii (GenBank AF406920) and $90 \%$ with Candida magnifica (GenBank U44821).
Table 1. Biochemical characteristics of Coccus Isolate AOD081EF from diseased Macrobrachium rosenbergii compared to characteristics of reference strains. AOD081EF: isolated from $M$. rosenbergii; EF: Enterococcus faecium CCRC 10067; EFC: E. faecalis CCRC 10066; ED: E. durans CCRC 10790;.EA: E.avium CCRC10801; MR1, Lactococcus garvieae isolated from $M$. rosenbergii; LG: $L$. garvieae ATCC 43921 (ATCC = American Type Culture Collection and CCRC = Culture Collection Research Center, Taiwan). RBC: red blood cells + : present; - : absent

\begin{tabular}{|c|c|c|c|c|c|c|c|}
\hline (API) Analytical profile index & AOD081EF & EF & EFC & ED & EA & MR1 & LG \\
\hline Pyruvate & + & + & + & + & + & + & + \\
\hline Hippurate & - & + & - & - & - & - & - \\
\hline Esculin & + & + & + & + & + & - & + \\
\hline Pyrrolldonyl 2-naphthylamide & + & + & + & + & + & + & + \\
\hline $\begin{array}{l}\beta \text {-bromo-2-naphthyl- } \alpha-\mathrm{D} \\
\text { galactopyranoside }\end{array}$ & + & + & - & + & - & - & - \\
\hline Naphthol-AS-BI $\beta$-D glucuronate & - & - & - & - & - & - & - \\
\hline 2.naphthyl- $\beta$-D D galactopyranoside & + & + & + & + & - & - & - \\
\hline 2.naphthyl phosphate & - & - & - & - & - & - & - \\
\hline L-leucine-2- naphthylamide & - & + & + & - & - & + & + \\
\hline Arginine & + & + & + & + & - & - & + \\
\hline Ribose & + & + & + & + & + & - & + \\
\hline L-arabinose & - & + & - & - & + & - & - \\
\hline Mannose & + & + & + & - & + & - & + \\
\hline Sorbitol & - & - & + & - & + & - & - \\
\hline Lactose & + & + & + & + & + & - & - \\
\hline Trehalose & + & + & + & - & + & - & + \\
\hline Inulin & - & - & - & - & + & - & - \\
\hline Raffinose & - & - & - & - & + & - & - \\
\hline Starch & - & - & + & + & + & - & - \\
\hline Glycogen & - & - & - & - & - & - & - \\
\hline Hemolysis (goat RBC) & $\gamma$ & $\gamma$ & $\gamma$ & $\alpha$ & $\alpha$ & $\alpha$ & $\alpha$ \\
\hline pH9.6 & + & + & + & + & + & + & + \\
\hline Tolerance at $6.0 \% \mathrm{NaCl}$ & + & + & + & + & + & - & + \\
\hline $6.5 \% \mathrm{NaCl}$ & + & + & + & - & + & - & + \\
\hline $8.0 \% \mathrm{NaCl}$ & + & + & + & - & - & - & - \\
\hline $10 \% \mathrm{NaCl}$ & - & - & - & - & - & - & - \\
\hline Growth at $4^{\circ} \mathrm{C}$ & + & + & + & + & + & + & + \\
\hline $10^{\circ} \mathrm{C}$ & + & + & + & + & + & + & + \\
\hline $15^{\circ} \mathrm{C}$ & + & + & + & + & + & + & + \\
\hline $25^{\circ} \mathrm{C}$ & + & + & + & + & + & + & + \\
\hline $37^{\circ} \mathrm{C}$ & + & + & + & + & + & + & + \\
\hline $40^{\circ} \mathrm{C}$ & + & + & + & + & + & + & + \\
\hline $45^{\circ} \mathrm{C}$ & + & + & + & + & + & - & + \\
\hline
\end{tabular}

\section{Experimental infection}

All prawns injected with $1.5 \times 10^{6}$ to $10^{7}$ of the coccus, yeast or mixture coccus and yeast died within $4 \mathrm{~d}$. The $\mathrm{LD}_{50}$ values for Coccus AOD081EF and Yeast AOD081MB isolates were $4.7 \times 10^{4}$ and $2.6 \times 10^{2} \mathrm{cfu}$ $\mathrm{ml}^{-1}$, respectively, while that of the bacterial-yeast coinfection was $2.4 \times 10^{2} \mathrm{cfu} \mathrm{ml}^{-1}$. Pure cultures of bacteria and yeast were re-isolated from the HP and muscle tissues of moribund prawns after challenge. No mortality or lesions occurred in the control group. Yellowishbrown body color, white muscle, milky hemolymph and histopathological changes similar to those in naturally infected prawns were observed except for the prawns injected with cocci only. These did not develop typical lesions but instead showed pathological changes similar to those seen in Lactococcus garvieae infection of the giant freshwater prawn (Chen et al. 2001). The coccus-yeast co-infection caused slightly higher mortality than did infections with either the coccus or yeast alone.

\section{DISCUSSION}

Muscle necrosis or spontaneous necrosis causing whitish muscle discoloration in decapod crustaceans is caused or exacerbated by inappropriate management and for unsuitable water parameters (e.g. handling, crowding; salinity, temperature, low dissolved oxygen: 

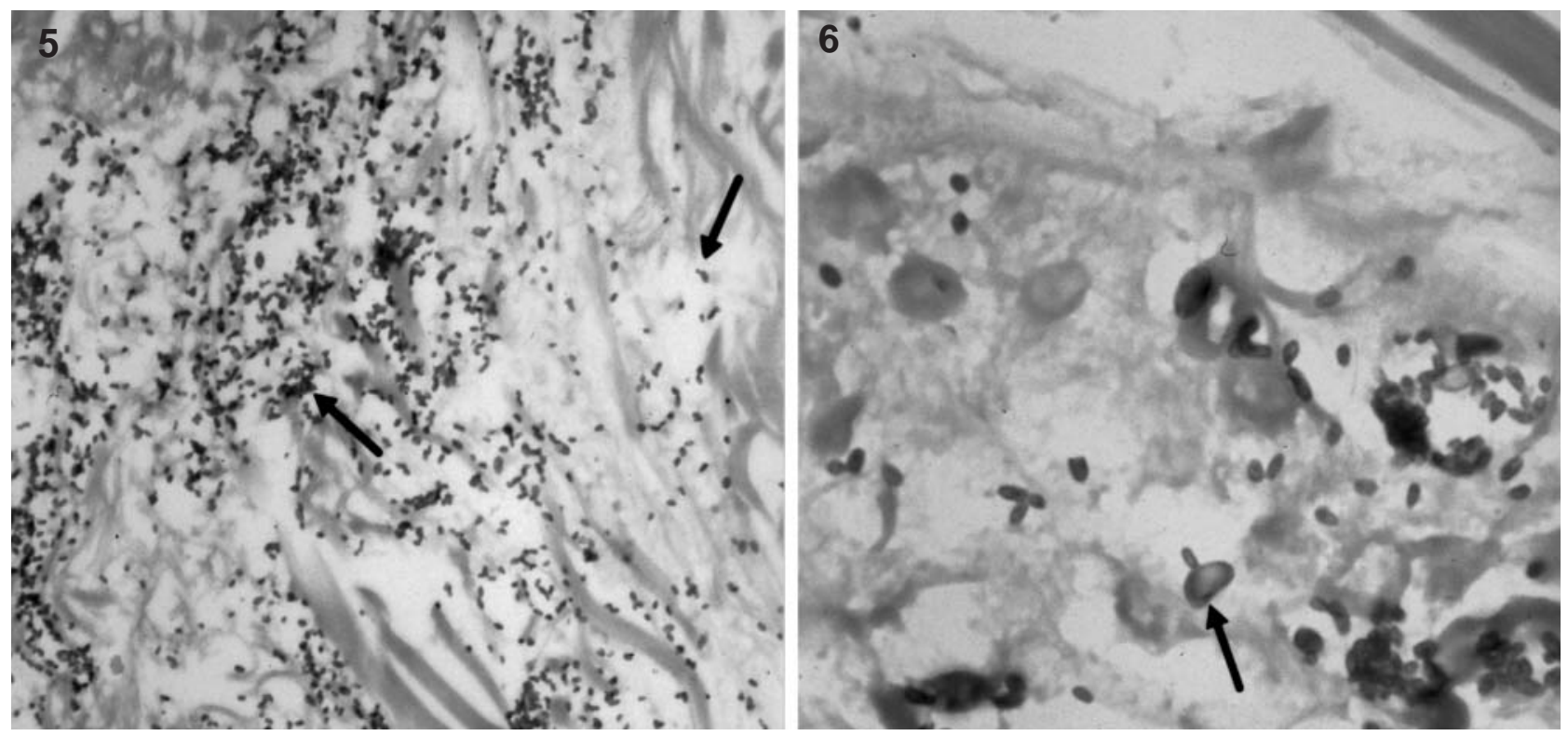

Figs. 5 \& 6. Macrobranchium rosenbergii. Fig. 5. Infected prawn muscle section showing accumulation of edematous fluid and necrotic areas with yeast microcolonies and single yeast cells (arrows) (PAS, $\times 200$ ). Fig. 6. Higher magnification of tissue in Fig. 5 showing typical yeast cells (arrows) in necrotic area $\overline{(\mathrm{PAS},} \times 1000)$

Lakshmi et al. 1978, Brock 1983, Lightner 1983,Cheng \& Chen 1998b,c). This opaque and whitish musculature discoloration commences at the tail and progresses to the head, which turns red (Lightner 1983). No bacterial cells are detectable. These changes are reversible during the initial stages of development if

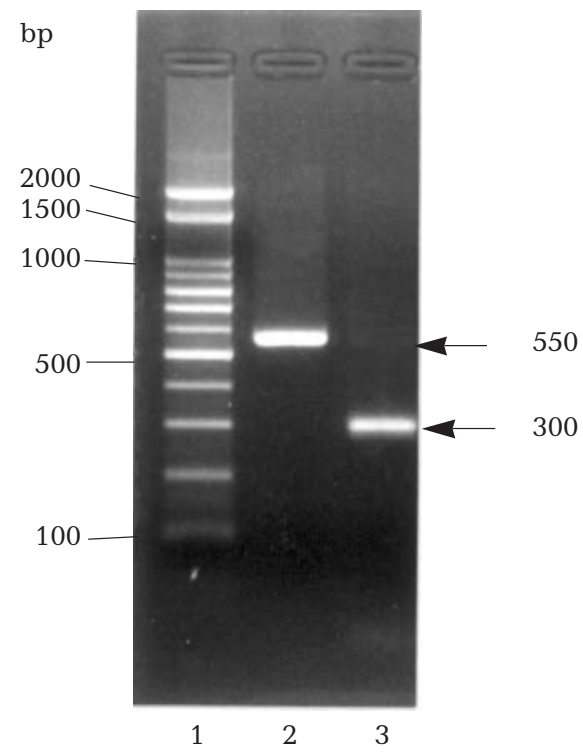

Fig. 7. Agarose gel of nested PCR products of D1/D2 domain of $26 \mathrm{~S}$ rDNA from Yeast Isolate AOD081MB. Step 1 gave 550 bp amplicon (Lane 2), Step 2 gave 300 bp amplicon (Lane 3). Lane 1: DNA molecular weight marker the causative stressor is eliminated. A similar phenomenon described by Cheng \& Chen (1998a) differed in that opaque and whitish muscle developed first in the cephalothorax and then progressed towards the abdominal muscle, where Enterococcus-like bacteria were present. In our study, the gross signs of disease showed some similarity to those described by Cheng \& Cheng (1998a) and to Lactococcus garvieae infections described by Chen et al. (2001) in Macrobranchium rosenbergii. However, the histopathology and gross signs in our study were different. The differences included a yellowish brown body color and marked necrotic areas, with yeast clumps and granulomas in several organs. Milky hemolymph has been described for $M$. rosenbergii yeast infections caused by Candida sake (I and II) (Lu et al. 1997, 1998).

The only Gram-positive coccus previously implicated in prawn infections was Lactococcus garviae (Chen et al. 2001). This was initially described as an Entrococcus-like pathogen (Cheng \& Chen 1998a) similar to E. seriolicida, which is known to be a pathogen of saltwater fish species (except eels) but has never been described in freshwater species (Kusuda et al. 1976, 1991) or outside Japan. Cheng \& Chen (1998a) reviewed the taxonomic changes involved in reducing E. seriolicida to a synonym of $L$. garvieae by genotypic methods (Eldar et al 1996).

For our coccus isolate AOD081EF, we used hemolysis, hippurate, salinity-tolerance $(6.5 \%)$, heat-tolerance $\left(45^{\circ} \mathrm{C}\right)$ and growth at $\mathrm{pH} 9.6$ as important criteria 
for streptococcus differentiation (Facklam \& Carey 1985, Schleifer \& Kilpper-Balz 1987, Murray 1990). According to these criteria, the morphology of the coccus and the results of physiological and biochemical tests on the API 20 Strep system, Coccus AOD081EF was identified as Entrococcus faecium. Our results were similar to those for the reference strain E. faecium CCRC 10067, except for hippurate, L-leucine-2 naththylamide, and L-arabinose. Despite these differences, we confirmed the identification by sequencing and comparison of $16 \mathrm{~S}$ rDNA gene sequences. A similar molecular comparison for the yeast isolate AOD081MB, but based on the D1/D2 domain of the 26S rDNA gene, indicated that this was Metschnikowia bicuspidata, which has not previously been reported as a prawn pathogen.

The results of our study on infected prawns from the field and on experimentally infected specimens constitute the first confirmation that co-infection by Enterococcus faecium and Metschnikowia bicuspidata can cause disease outbreaks in the giant freshwater prawn Macrobrachium rosenbergii.

Acknowledgements. The authors thank Dr. Terutoyo Yoshida, Miyazaki University, Miyazaki, Japan, for supplying the bacterial isolate Lactococcus garvieae MZ9101. This study was supported by the National Science Council, Taiwan, ROC (grants NSC91-2313-B020-025 and NSC91-2313-B020-028).

\section{LITERATURE CITED}

Brock JA (1983) Diseases (infectious and noninfectious) metazoan parasites, predator, and public health considerations in Macrobrachium culture and fisheries. In McVey JP (ed) CRC handbook of mariculture, Vol 1. Crustacean aquaculture. CRC Press, Boca Raton, FL, p 329-370

Chen SC, Lin, YD, Liaw LL, Wang PC (2001) Lactococcus garvieae infection in the giant freshwater prawn Macrobrachium rosenbergii confirmed by polymerase chain reaction and $16 \mathrm{~S}$ rDNA sequencing. Dis Aquat Org 45:45-52

Cheng W, Chen JC (1998a) Isolation and characterization of an Enterococcus-like bacterium causing muscle necrosis

Editorial responsibility: Timothy Flegel,

Bangkok, Thailand and mortality in Macrobrachium rosenbergii in Taiwan. Dis Aquat Org 34:93-101

Cheng W, Chen JC (1998b) Enterococcus-like infections in Macrobrachium rosenbergii are exacerbated by high $\mathrm{pH}$ and temperature but reduced by low salinity. Dis Aquat Org 34:103-108

Cheng W, Chen JC (1998c) Effect of cultivation broth $\mathrm{pH}$ temperature and $\mathrm{NaCl}$ concentration on virulence of Enterococcus-like bacterium to the giant fresh prawn Macrobrachium rosenbergii. Dis Aquat Org 36:233-237

Eldar AC, Ghittino C, Asanta L, Bvozzettz E, Goria M (1996) Enterococcus seriolicida is a junior synonym of Lactococcus garvieae, a causative agent of septicemia and meningoencephalitis in fish. Curr Microbiol 32:85-88

Facklam RR, Carey RB (1985) Manual of clinical microbiology, 4th edn. In: Lennette EH, Ballows A, Hausler WJ Jr, Shadomy HJ (eds) Streptococci and aerococci. American Society for Microbiology, Washington, DC, p 154-175

Kurtzman CP, Robnett CJ (1997) Identification of clinically important ascomycetous yeasts based on nucleotide divergence in the $5^{\prime}$ end of the large-subunit (26S) ribosomal DNA gene. J Clin Microbiol 35:1216-1223

Kusuda R, Kawai K, Salati F, Banner CR, Fryer JL (1991) Enterococcus seriolicida sp. nov., a fish pathogen. Int J Syst Bacteriol 41:406-409

Kusuda R, Kawai K, Toyoshima T, Komatsu I (1976) A new pathogenic bacterium belonging to genus Streptococcus isolated from an epizootic of cultured yellowtail. Bull Jpn Soc Sci Fish 42:1345-1352

Lakshmi GJ, Venkataramiam A, Howse HD (1978) Effect of salinity and temperature changes on spontaneous muscle necrosis in Penaeus aztecus. Aquaculture 13:35-43

Lightner DV (1983) Diseases of culture penaeid shrimp. In: McVey JP (ed) CRC handbook of mariculture, Vol 1. Crustacean aquaculture. CRC Press, Boca Raton, FL, p 289-320

Lu CC, Tang FJ, Yoichiro U, Kou GH, Chen SN (1997) Yeast infection in prawns (Macrobrachium rosenbergii de Man) in Taiwan. Acta Zool Taiwanica 8:33-45

Lu CC, Tang KFG, Chen SN (1998) identification and genetic characterization of yeast isolated from freshwater prawns, Macrobrachium rosenbergii de Man, in Taiwan. J Fish Dis 21:185-192

Murray BB (1990) The life and times of the Enterococcus. Clin Microbiol Rev 3:46-65

New MB (1995) Status of freshwater prawn farming: a review. Aquac Res 3:46-65

Schleifer KH, Kilpper-Baz R (1987) Molecular and chemotaxnomic approaches to the classification of streptococci, enterococci and lactococci: a review system. Appl Microbiol 10:1-9

Submitted: August 16, 2002; Accepted: February 15, 2003 Proofs received from author(s): June 23, 2003 